\title{
Examining Nonstandard Higgs Couplings via Photon-Photon Fusion at FCC
}

\author{
Gülistan AKKAYA SELÇİN* \\ Bitlis Eren University, Faculty of Science, Physics Department, Bitlis \\ (ORCID: 0000-0003-4720-6631)
}

\begin{abstract}
In this paper, the constraints on the non-standard $\mathrm{HZ} \gamma$ and $\mathrm{H} \gamma \gamma$ couplings through the exclusive single Higgs production process $\mathrm{pp} \rightarrow \mathrm{p} \gamma \gamma \mathrm{p} \rightarrow \mathrm{pHp}$ at a future $100 \mathrm{TeV}$ proton-proton collider have been examined. As the signal, $\mathrm{H} \rightarrow \gamma \gamma$ and $\mathrm{H} \rightarrow \mathrm{Z} \gamma$ decay channels of the Higgs boson have been considered. Taking into account three different acceptance regions of $0.015<\xi<0.15,0.0015<\xi<0.5,0<\xi<1-\frac{m_{p}}{E}$ and integrated luminosity of $L_{\text {int }}=$ $3000 \mathrm{fb}^{-1}$, it has been found $95 \%$ C.L. sensitivity constraints on these couplings. Similar calculations have been made at the LHC and discussed the improvement which is provided by a future $100 \mathrm{TeV}$ proton-proton collider with respect to $\mathrm{LHC}$.
\end{abstract}

Key words: Higgs couplings, single Higgs production, $100 \mathrm{TeV}$ proton-proton collider.

\section{FCC'de Foton-Foton Füzyonu Yoluyla Anormal Higgs Bağlaşımları'nın İncelenmesi}

\section{Öz}

$\mathrm{Bu}$ çalışmada, $100 \mathrm{TeV}$ proton-proton çarpıştırıcısında, harici tek Higgs üretimi olan pp $\rightarrow$ p $\gamma \gamma \mathrm{p} \rightarrow \mathrm{pHp}$ süreci üzerinden anormal $\mathrm{HZ} \gamma$ ve $\mathrm{H} \gamma \gamma$ bağlaşımlarının sınırları incelenmiştir. Sinyal olarak, Higgs bozonunun $\mathrm{H} \rightarrow \gamma \gamma$ ve $\mathrm{H} \rightarrow \mathrm{Z} \gamma$ bozounum kanalları ele alınmıştır. Üç farklı akseptans bölgesi $0.015<\xi<0.15 ; 0.0015<\xi<0.5 ; 0<\xi<1-$ $\frac{m_{p}}{E}$ ve integre edilmiş luminosite $L_{\text {int }}=3000 \mathrm{fb}^{-1}$ değerleri dikkate alınarak, bu bağlaşımların \%95 güvenilirlik düzeyindeki duyarlılık limitleri bulunmuştur. Aynı hesaplar LHC için de yapılmış ve gelecekte kurulması planlanan $100 \mathrm{TeV}$ proton-proton çarpıştırıcısının LHC'ye göre sağladığı iyileştirme yorumlanmıştır.

Anahtar kelimeler: Higgs bağlaşımları, tek Higgs üretimi, $100 \mathrm{TeV}$ proton-proton çarpıştırıcısı.

\section{Introduction}

The physics potential of a future $100 \mathrm{TeV}$ proton-proton collider has been discussed recently by the physics community and the interest in the subject is growing rapidly [1-3]. Such a very high energetic machine has a great potential to probe the new physics. The predictions of the SM have been confirmed by previous and present experiments. The last great confirmation of the SM is the discovery of the Higgs boson $[4,5]$. The next step is to study the properties of Higgs boson and its couplings to other SM particles. These studies will confirm the SM and will be useful for new physics beyond SM. The future $100 \mathrm{TeV}$ proton-proton collider provides an ideal venue to measure non-standard couplings of the Higgs boson. Phenomenological studies on non-standard Higgs couplings in a future $100 \mathrm{TeV}$ collider have been rapidly growing in the literature [1,3,6-12]. In this article we consider exclusive single Higgs production process $\mathrm{pp} \rightarrow \mathrm{p} \gamma \gamma \mathrm{p} \rightarrow \mathrm{pHp}$ in a $100 \mathrm{TeV}$ proton-proton collider. A schematic diagram of this reaction is given in Figure 1.

\footnotetext{
*Sorumlu yazar: gakkaya@beu.edu.tr

Geliş Tarihi: 15.10.2019, Kabul Tarihi: 19.12.2019
} 


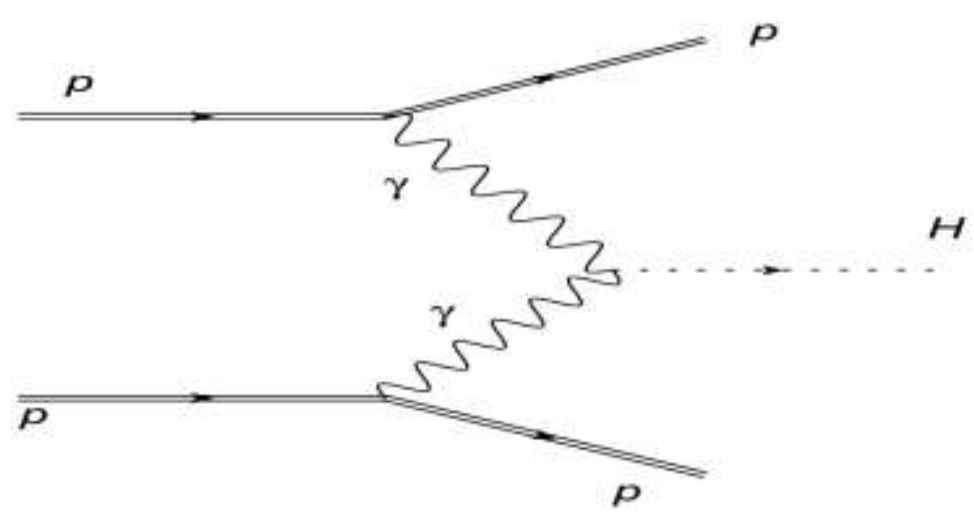

Figure 1. Exclusive single Higgs boson production through photon-photon fusion in a pp collision.

In such a reaction, incoming protons emit two quasi-real photons which have a very low virtuality and then these photons interact each other to produce a Higgs boson. Initial protons do not dissociate into partons becouse the virtuality of emitted photons are very low. This production provide a very clean experimental environment which is free from serious uncertainties coming from parton jets, because there aren't any proton remnants. This circumstance makes it easy to detect the signal that we want to measure. Furthermore, photon-induced processes are electromagnetic in nature, free from complicated QCD backgrounds. The exclusive production was studied experimentally at the Large Hadron Collider (LHC) and also at the former hadron collider Fermilab Tevatron [13-24]. It was verified that complementary to deep inelastic hadron collisions, photon-induced processes via equivalent photons can be studied in a hadron collider. Therefore, it is interesting to investigate such photoninduced processes in the future proton collider.

\section{The cross section and non-standard $\mathrm{HZ} \gamma$ and $\mathrm{H} \gamma \gamma$ couplings}

The single Higgs can be produced through the interaction of two equivalent photons emitted from the

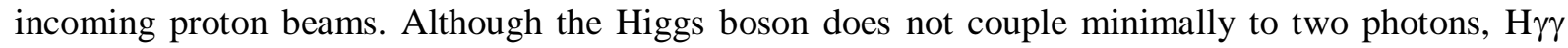
vertex gets SM contributions from one-loop level. There may be also new physics contributions which modify this vertex. Hence, in principle these new physics contributions can be probed by measuring the production cross section. However, Higgs bosons cannot be detected directly by the detectors. It can be detected from its decay products. Final-state particles' invariant mass measurement will confirm the existence of on-mass-shell Higgs bosons. As the signal, we consider $\mathrm{H} \rightarrow \gamma \gamma$ and $\mathrm{H} \rightarrow \mathrm{Z} \gamma$ decay channels of the Higgs boson. Taking into account the narrow width approximation for the processes $\gamma \gamma \rightarrow \mathrm{H} \rightarrow \mathrm{Z} \gamma$ and $\gamma \gamma \rightarrow \mathrm{H} \rightarrow \gamma \gamma$ the cross section can be calculated easily:

$$
\begin{aligned}
& \sigma(\gamma \gamma \rightarrow \mathrm{H} \rightarrow \gamma \gamma)=\frac{8 \pi^{2}}{m_{H}} \frac{\Gamma_{\gamma}{ }^{2}}{\Gamma_{\text {total }}} \delta\left(s-m_{H}^{2}\right) \\
& \sigma(\gamma \gamma \rightarrow \mathrm{H} \rightarrow \mathrm{Z} \gamma)=\frac{8 \pi^{2}}{m_{H}} \frac{\Gamma_{\gamma} I \mathrm{Z}_{\gamma}}{\Gamma_{\text {total }}} \delta\left(s-m_{H}^{2}\right)
\end{aligned}
$$

where $\Gamma_{\mathrm{z} \gamma}$ and $\Gamma_{\gamma \gamma}$ represent the decay width of $\mathrm{H} \rightarrow \mathrm{Z} \gamma$ and $\mathrm{H} \rightarrow \gamma \gamma$ respectively, $\Gamma_{\text {tot }}$ is the Higgs boson's total decay width, and $s$ is the Mandelstam parameter of the initial state photon pair. During numerical calculations, Higgs boson's total decay width is taken to be $\Gamma_{\text {tot }}=6.1 \mathrm{MeV}$ [25]. The total cross section of the main process $\mathrm{pp} \rightarrow \mathrm{p} \gamma \gamma \mathrm{p} \rightarrow \mathrm{pHp} \rightarrow \mathrm{p} \gamma \gamma(\mathrm{Z} \gamma) \mathrm{p}$ in the proton-proton collision can be obtained by integrating the cross section for sub-processes over the equivalent photon distribution functions:

$$
\begin{aligned}
& \sigma(\mathrm{pp} \rightarrow \mathrm{p} \gamma \gamma \mathrm{p} \rightarrow \mathrm{pHp} \rightarrow \mathrm{p} \gamma \gamma(\mathrm{Z} \gamma) \mathrm{p})= \\
& \quad \int_{x_{\min }}^{x_{\max }} d x_{1} \int_{x_{\min }}^{x_{\max }} d x_{2} f_{\gamma}\left(x_{1}\right) f_{\gamma}\left(x_{2}\right) \sigma(\gamma \gamma \rightarrow \mathrm{H} \rightarrow \gamma \gamma(\mathrm{Z} \gamma))
\end{aligned}
$$


Here, $f_{\gamma}\left(x_{1(2)}\right)$ is the equivalent photon distribution function, $x_{1(2)}$ is the fraction that denotes the ratio between the emitted equivalent photon and incoming proton energies ; $x_{1(2)}=\frac{E_{\gamma_{1(2)}}}{E_{p}}$. After appropriate change of variables and using the Dirac delta functional in the cross sections Eq.(1) and Eq.(2), the two-dimensional integral in Eq.(3) is reduced to the following one-dimensional integral:

$$
\begin{aligned}
\sigma(\mathrm{pp} \rightarrow \mathrm{p} \gamma \gamma \mathrm{p} \rightarrow \mathrm{pHp} \rightarrow \mathrm{p} \gamma \gamma(\mathrm{Z} \gamma) \mathrm{p})=\frac{8 \pi^{2}}{m_{H}} \frac{\Gamma_{\gamma}{ }^{2}\left(\Gamma_{\mathrm{z} \gamma} / \Gamma_{\gamma \gamma}\right)}{\Gamma_{\text {total }}} \\
\quad \times \int_{\operatorname{Max}\left(\frac{m_{H}{ }^{2}}{4 E_{p} x_{\max }}, E_{p} x_{\min }\right)}^{\frac{d y}{4 y} x_{\max }} f_{\gamma}(\mathrm{y}) f_{\gamma}\left(\frac{m_{H}{ }^{2}}{4 y}\right)
\end{aligned}
$$

Here, $y$ is the one of the equivalent photon's energy. The $x$ parameter's lower and upper bounds can be found easily from the kinematical constraints; $0<x<1-\frac{m_{p}}{E_{p}}$. At high energetic collisions the $\mathrm{x}$ parameter can be assumed to be equal to the momentum fraction loss $\xi=\left(|\vec{p}|-\left|\vec{p}^{\prime}\right|\right) / \vec{p}$ of the intact scattered protons. In this formula, $\vec{p}$ represents the incoming proton's momentum and $\vec{p}$ 'represents the intact scattered proton's momentum. After emission of the elastic photon, protons deviate lightly from the beam direction and can't be detected by the central detectors. The lost energy signature that arises from this situation called the large rapidity gap. The large rapidity region's lost energy signature is used to identify the exclusive processes. Furthermore, very forward detectors can detect the intact scattered protons. The detection of intact protons in the very forward detectors provides an accurate identification of an exclusive process. The LHC is designed to equip with very forward detectors $[15,16,26]$. We assume that a similar very forward detector equipment will be installed on the future proton-proton collider. The very forward detectors have a limited range. They cannot detect all scattered protons in the kinematically allowed interval, $0<\xi<1-\frac{m_{p}}{E_{p}}$. The upper and lower limits of forward detector's ranges which called the forward detector acceptances are $\xi_{\max }$ and $\xi_{\min }$ respectively. During numerical calculations, in addition to the whole region $0<\xi<1-\frac{m_{p}}{E_{p}}$, we will take into account forward detector acceptances of $0.0015<\xi<0.5$ and $0.015<\xi<0.15$. In this paper we work in a model independent framework and concern with non-standard Higgs couplings to gauge bosons. Thus we take into account the total effective lagrangian as follows [27-34]:

$$
\mathcal{L}_{e f f}=\mathcal{L}_{S M}+\sum_{n} \frac{f_{n}}{\Lambda^{2}} \mathcal{O}_{n}+\ldots
$$

Here, the first term is the SM lagrangian of dimension four and the second term constitutes the contribution of new physics which deviations in the $H V_{1} V_{2}$ couplings $\left(V_{i}=W, Z, \gamma\right)$ from SM predictions. Also, $\mathcal{O}_{n}$ 's are the operators that consist of Higgs-boson fields or vector-boson, $\Lambda$ is the scale of new physics and $f_{n}$ denote non-standard couplings Taking into account the $\mathcal{O}_{n}$ operators to be $\mathrm{P}$ and $\mathrm{C}$ even, there are five dimension- six operators that alter the Higgs boson couplings to $\gamma$ and $\mathrm{Z}$. The exact expressions of these operators can be found in [27, 28, 34]. They are given as:

$$
\begin{aligned}
& \mathrm{O}_{\mathrm{ww}}=\phi^{\mathrm{t}} \mathrm{W} \mu \nu \mathrm{W}^{\mu v} \phi \\
& \mathrm{O}_{\mathrm{W}}=\left(\mathrm{D}_{\mu} \phi\right)^{+} \mathrm{W}^{\mu v}\left(\mathrm{D}_{v} \phi\right) \\
& \mathrm{O}_{\mathrm{BB}}=\phi^{\mathrm{t}} \mathrm{B}_{\mu v} \mathrm{~B}^{\mu v} \phi \\
& \mathrm{O}_{\mathrm{B}}=\left(\mathrm{D}_{\mu} \phi\right)^{+} \mathrm{B}^{\mu v}\left(\mathrm{D}_{v} \phi\right) \\
& \mathrm{O}_{\mathrm{BW}}=\phi^{\mathrm{t}} \mathrm{B}_{\mu v} \mathrm{~W}^{\mu v} \phi
\end{aligned}
$$

where $\phi$ is the scalar doublet, $D_{\mu}$ is the covariant derivative, $W_{\mu \nu}=i \frac{g}{2}\left(\vec{\sigma} \cdot \overrightarrow{W_{\mu \nu}}\right)$ and $\mathrm{B}_{\mu \nu}=i \frac{g^{\prime}}{2} \overrightarrow{B_{\mu \nu}}$. Here $\mathrm{g}$ and $g^{\prime}$ are the $\mathrm{S} U(2)_{L}$ and $U(1)_{Y}$ gauge couplings. And $\sigma$ are the pauli matrices. 
After electroweak symmetry breaking the effective lagrangian written for the new physics in Eq. (5) is expressed with the physical fields. Taking into account $\mathrm{HZ} \gamma$ and $\mathrm{H} \gamma \gamma$ vertices, it can be expressed as follows:

$$
\mathcal{L}_{e f f}^{(N P)}=g_{H \gamma \gamma} H A_{\mu \nu} A^{\mu \nu}+g_{H Z \gamma}^{(1)} A_{\mu \nu} Z^{\mu} \partial^{v} H+g_{H Z \gamma}^{(2)} H A_{\mu \nu} Z^{\mu \nu}
$$

where $V_{\mu \nu}=\partial_{\mu} V_{\nu}-\partial_{\nu} V_{\mu}$ with $\mathrm{Z}$ field and $\mathrm{V}=\mathrm{A}$ (photon) $. g_{H \gamma \gamma}, g_{H \mathrm{Z} \gamma}^{(1)}$ and $g_{H \mathrm{Z} \gamma}^{(2)}$ are the non-standard couplings which involve $f_{n}$ appearing in the effective lagrangian (5) before symmetry breaking as;

$g_{H \gamma \gamma}=-\left(\frac{g_{m w}}{\Lambda^{2}}\right) \sin \theta_{w}^{2}\left(\frac{f_{B B+} f_{w w-} f_{B w}}{2}\right)$

$g_{H Z \gamma}^{1}=\left(\frac{g_{m w}}{\Lambda^{2}}\right) \sin \theta_{w}\left(\frac{f_{w}-f_{B}}{2 \cos \theta_{w}}\right)$

$g_{H Z \gamma}^{2}=\left(\frac{g_{m w}}{\Lambda^{2}}\right) \frac{\sin \theta_{w}}{2 \cos \theta_{w}}\left[2 \sin \theta_{w}^{2} f_{B B-} 2 \cos \theta_{w}^{2} f_{w w}+\left(\cos \theta_{w}^{2}-\sin \theta_{w}^{2}\right) f_{B w}\right]$

where $\mathrm{s}=\sin \theta_{w}, \mathrm{c}=\cos \theta_{w}, \theta_{w}$ is the Weinberg angle and $m_{w}$ is the mass of the W boson.

$\mathrm{H} \gamma \gamma$ and $\mathrm{HZ} \gamma$ vertices disappear at the tree-level in the SM but they appear at one-loop level. At high energies $\left(\sqrt{s} \gg m_{H}\right)$ these one-loop contributions can be expressed with the following effective lagrangian $[35,36]$ :

$$
\mathcal{L}_{e f f}^{(S M)}=g_{H \gamma \gamma}^{(S M)} H A_{\mu \nu} A^{\mu \nu}+g_{H Z \gamma}^{(S M)} H A_{\mu \nu} Z^{\mu \nu}
$$

where, $g_{H \gamma \gamma}^{(S M)}=\frac{2 \alpha}{9 \pi v}$ and $g_{H \mathrm{Z} \gamma}^{(S M)}=\frac{\alpha}{4 \pi v \sin \theta_{W}}(5.508-0.004 i)$. Here, $v$ is the electroweak vacuum expectation value and $\alpha$ is the fine structure constant. Therefore during the calculations we consider;

$\mathcal{L}_{\text {eff }}^{\prime}=\mathcal{L}_{\text {eff }}^{(S M)}+\mathcal{L}_{\text {eff }}^{(N P)}=g_{H \gamma \gamma}^{\prime} H A_{\mu \nu} A^{\mu \nu}+g_{H \mathrm{Z} \gamma}^{\prime(1)} A_{\mu \nu} Z^{\mu} \partial^{\nu} H+g_{H \mathrm{Z} \gamma}^{\prime(2)} H A_{\mu \nu} Z^{\mu \nu}$

where

$$
\begin{aligned}
& g_{H \gamma \gamma}^{\prime}=g_{H \gamma \gamma}+g_{H \gamma \gamma}^{(S M)} \\
& g_{H Z \gamma}^{\prime(1)}=g_{H Z \gamma}^{(1)} \\
& g_{H Z \gamma}^{(2)}=g_{H Z \gamma}^{(2)}+g_{H Z \gamma}^{(S M)}
\end{aligned}
$$

The decay width of $\mathrm{H} \rightarrow \mathrm{Z} \gamma$ and $\mathrm{H} \rightarrow \gamma \gamma$ can be expressed in the terms of $g_{H \gamma \gamma}^{\prime}, g_{H \mathrm{Z} \gamma}^{\prime(1)}$ and $g_{H \mathrm{Z} \gamma}^{\prime(2)}$ as follows:

$$
\begin{aligned}
& \Gamma_{\gamma \gamma}=\frac{g_{\mathrm{H} \gamma}^{\prime 2} m_{H}^{3}}{4 \pi} \\
& \Gamma_{\mathrm{Z} \gamma}=\left(g_{\mathrm{H} \mathrm{z} \gamma}^{\prime(1)}+2 g_{\mathrm{H} \mathrm{z} \gamma}^{\prime(2)}\right)^{2} \frac{\left(m_{\left.\mathrm{H}-m_{\mathrm{Z}}^{2}\right)^{3}}^{32 \pi m_{\mathrm{H}}^{3}}\right.}{3}
\end{aligned}
$$

In Figure 2- Figure 4, taking into account $\mathrm{H} \rightarrow \mathrm{Z} \gamma$ decay of the produced Higgs bosons, the cross section of main process $\mathrm{pp} \rightarrow \mathrm{p} \gamma \gamma \mathrm{p} \rightarrow \mathrm{pHp} \rightarrow \mathrm{pZ} \gamma \mathrm{p}$ is plotted as a function of non-standard couplings $g_{H \gamma \gamma}, g_{H \mathrm{Z} \gamma}^{(1)}$ and $g_{H \mathrm{Z} \gamma}^{(2)}$ for the kinematically allowed interval of $0<\xi<1-\frac{m_{p}}{E_{p}}$ and for $0.015<\xi$ $<0.15$ and $0.0015<\xi<0.5$. 


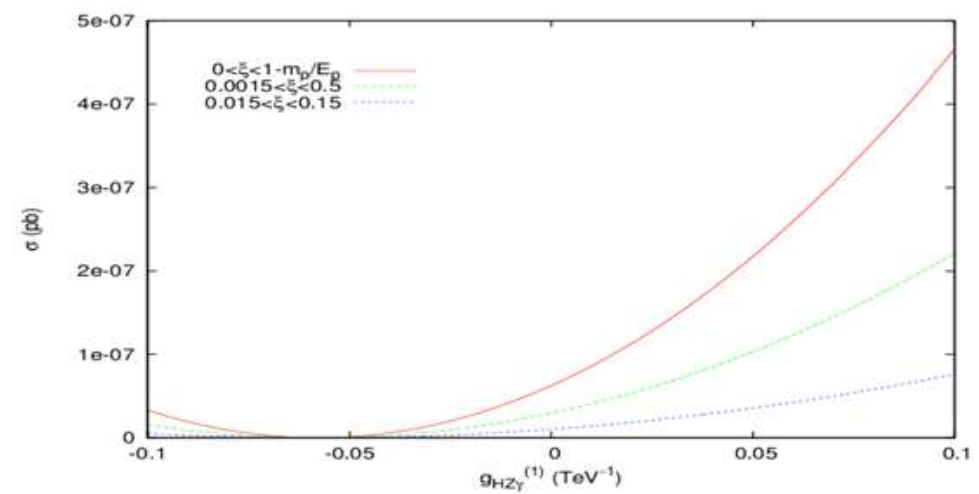

Figure 2. The cross section of $\mathrm{pp} \rightarrow \mathrm{p} \gamma \gamma \mathrm{p} \rightarrow \mathrm{pHp} \rightarrow \mathrm{pZ} \gamma \mathrm{p}$ as a function of the non-standard $g_{H \mathrm{Z} \gamma}^{(1)}$ coupling at a 100 $\mathrm{TeV}$ collider. Other non-standard couplings are assumed to be zero.

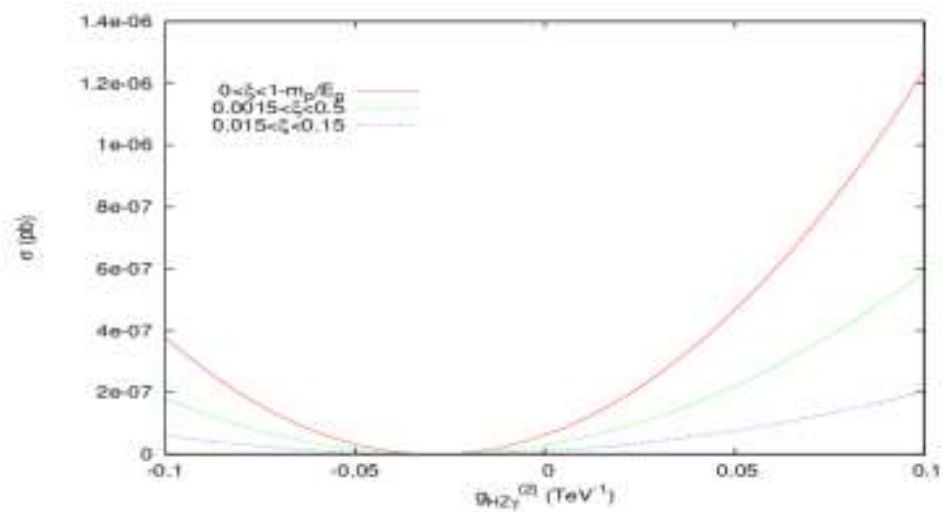

Figure 3. The cross section of $\mathrm{pp} \rightarrow \mathrm{p} \gamma \gamma \mathrm{p} \rightarrow \mathrm{pHp} \rightarrow \mathrm{pZ} \gamma \mathrm{p}$ as a function of the non-standard $g_{H \mathrm{Z} \gamma}^{(2)}$ coupling at a $100 \mathrm{TeV}$ collider. Other non-standard couplings are assumed to be zero.

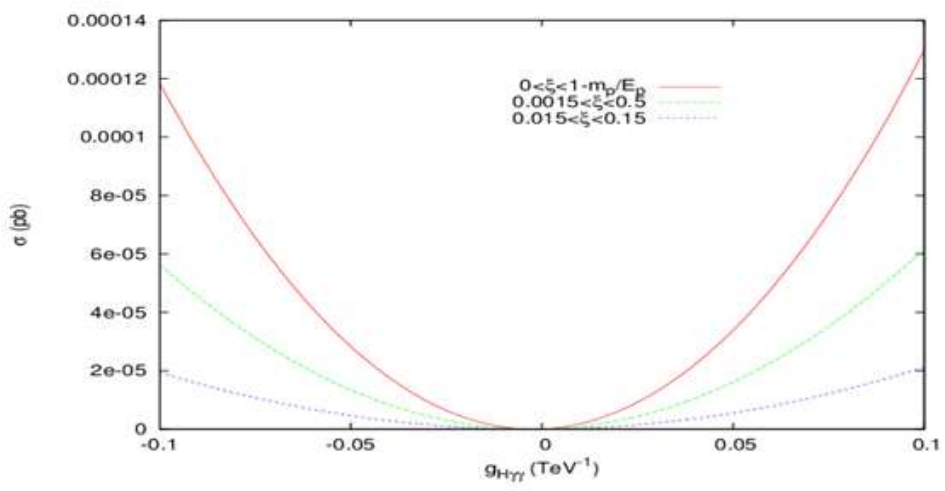

Figure 4. The cross section of $\mathrm{pp} \rightarrow \mathrm{p} \gamma \gamma \mathrm{p} \rightarrow \mathrm{pHp} \rightarrow \mathrm{pZ} \gamma \mathrm{p}$ as a function of the non-standard $g_{H \gamma \gamma}$ coupling at a 100 TeV collider. Other non-standard couplings are assumed to be zero.

Similarly, in Figure 5, taking into account $\mathrm{H} \rightarrow \gamma \gamma$ decay and the same acceptances mentioned above, we plot the cross section of main process $\mathrm{pp} \rightarrow \mathrm{p} \gamma \gamma \mathrm{p} \rightarrow \mathrm{pHp} \rightarrow \mathrm{p} \gamma \gamma \mathrm{p}$ as a function of non-standard $g_{H \gamma \gamma}$ coupling. 


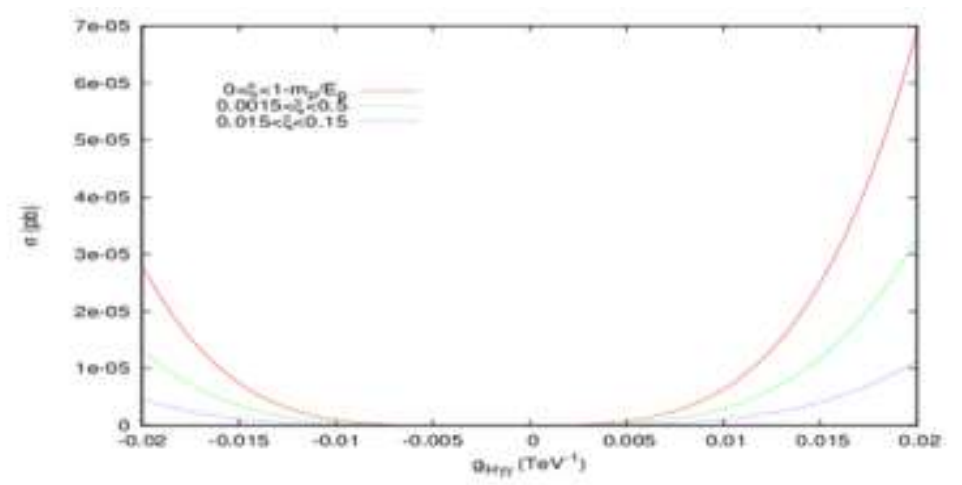

Figure 5. The cross section of $\mathrm{pp} \rightarrow \mathrm{p} \gamma \gamma \mathrm{p} \rightarrow \mathrm{pHp} \rightarrow \mathrm{p} \gamma \gamma \mathrm{p}$ as a function of the non-standard $g_{H \gamma \gamma}$ coupling at a 100 TeV collider. Other non-standard couplings are assumed to be zero.

In Figure 2- Figure 5, the cross section for the whole region $\left(0<\xi<1-\frac{m_{p}}{E_{p}}\right)$ increases more rapidly as a function of non-standard coupling comparing with the those for forward detector acceptances. This is reasonable since, the initial $\gamma \gamma$ system's center-of-mass energy increases as the $\xi$ interval increases. In order to obtain a definite result we perform a statistical analysis. We use Poisson analysis because the SM events' number is less than 1. Then we obtain 95\% C.L. sensitivity constraints on non-standard coupling parameters. For the number of events we use the formula $N=\sigma \times L_{\text {int }}$,

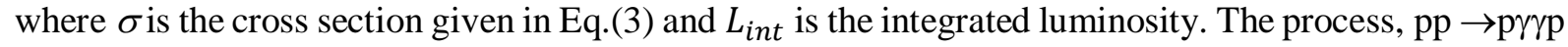
$\rightarrow \mathrm{pHp} \rightarrow \mathrm{p} \gamma \gamma \mathrm{p}$ receives contributions only $\mathrm{H} \gamma \gamma$ from vertex. Therefore, in this case only non-standard $g_{H \gamma \gamma}$ coupling is restricted. The corresponding bounds on $g_{H \gamma \gamma}$ is given in Figure 6 for future $100 \mathrm{TeV}$ proton-proton collider with $L_{\text {int }}=3000 \mathrm{fb}^{-1}$.
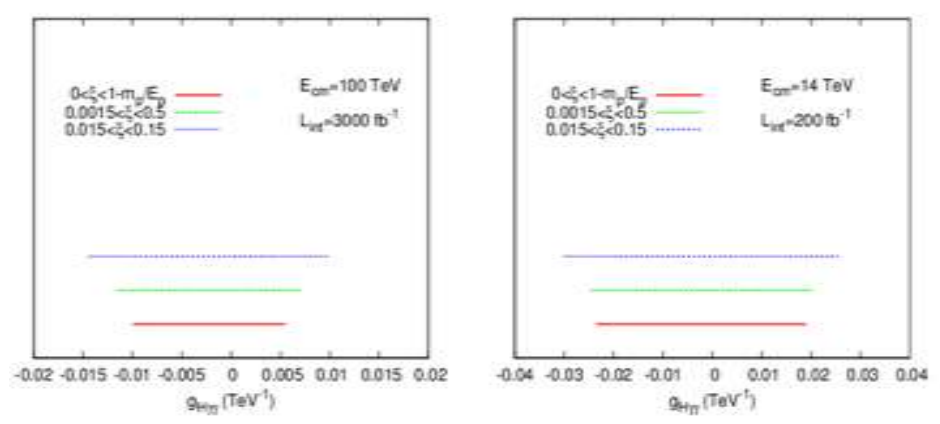

Figure 6. In the left panel $95 \%$ C.L. sensitivity constraints on the coupling $g_{H \gamma \gamma}$ are given for the $\sqrt{s}=$ $100 \mathrm{TeV}$ and $L_{\text {int }}=3000 \mathrm{fb}^{-1}$. In the right panel, for the $\operatorname{LHC}\left(\sqrt{\mathrm{s}}=14 \mathrm{TeV}, L_{\text {int }}=200 \mathrm{fb}^{-1}\right)$ similar constraints are given. $\mathrm{H} \rightarrow \gamma \gamma$ decay channel is considered as the signal.

This luminosity value has been proposed for future $100 \mathrm{TeV}$ proton-proton collider and used in similar studies. For example, see [1]. For a comparison, we do a similar analysis for the $\operatorname{LHC}(\sqrt{s}=$ $\left.14 \mathrm{TeV}, L_{\text {int }}=200 \mathrm{fb}^{-1}\right)$. However, the process $\mathrm{pp} \rightarrow \mathrm{p} \gamma \gamma \mathrm{p} \rightarrow \mathrm{pHp} \rightarrow \mathrm{pZ} \gamma \mathrm{p}$ receives contributions both from $\mathrm{H} \gamma \gamma$ and $\mathrm{HZ} \gamma$ vertices. Therefore, in this case we have to analyze three independent couplings $g_{H \gamma \gamma}, g_{H Z \gamma}^{(1)}$ and $g_{H Z \gamma}^{(2)}$. For simplicity, we assume that only two of the couplings are free but the remaining one is zero. In Figure 7- Figure 9, we present 95\% C.L. constraints on $g_{H \mathrm{Z} \gamma}^{(2)}-g_{H \gamma \gamma}, g_{H \mathrm{Z} \gamma}^{(1)}-$ $g_{H \gamma \gamma}$ and $g_{H Z \gamma}^{(2)}-g_{H Z \gamma}^{(1)}$ respectively. 

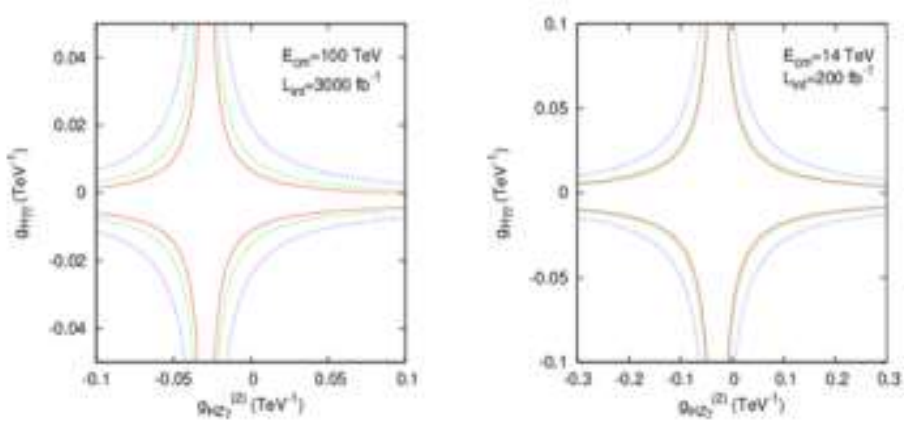

Figure 7. The $95 \%$ C.L. sensitivity constraints on $g_{\mathrm{HZ} \gamma}^{(2)}-g_{H \gamma \gamma}$ for $100 \mathrm{TeV}$ pp collider (left panel) and LHC (right panel). $\mathrm{H} \rightarrow \mathrm{Z} \gamma$ decay channel is considered as the signal. The solid line shows the bounds for the whole interval $0<\xi<1-\frac{m_{p}}{E_{p}}$. The dotted and dashed lines indicate the bounds for $0.015<\xi<0.15$ and $0.0015<\xi<0.5$, respectively.
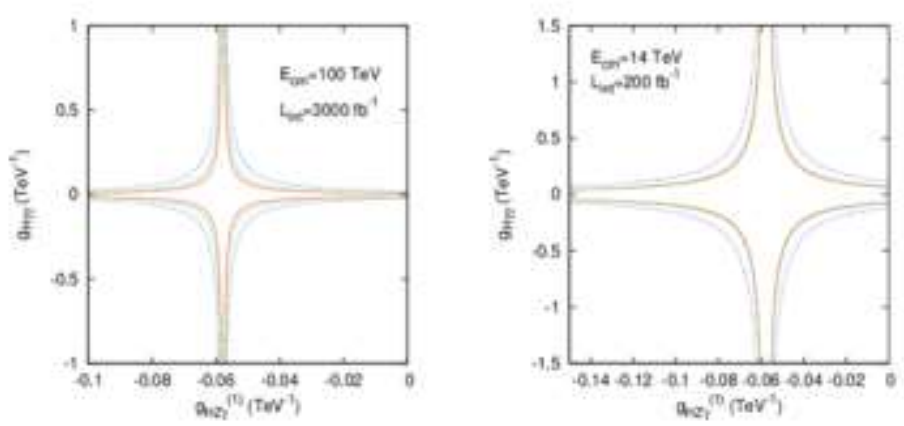

Figure 8. The $95 \%$ C.L. sensitivity constraints on $g_{H Z_{\gamma}}^{(1)}-g_{H \gamma \gamma}$ for $100 \mathrm{TeV}$ pp collider (left panel) and LHC (right panel). $\mathrm{H} \rightarrow \mathrm{Z} \gamma$ decay channel is considered as the signal. The solid line shows the bounds for the whole interval $0<\xi<1-\frac{m_{p}}{E_{p}}$. The dotted and dashed lines indicate the bounds for $0.015<\xi<0.15$ and $0.0015<\xi<0.5$ respectively.
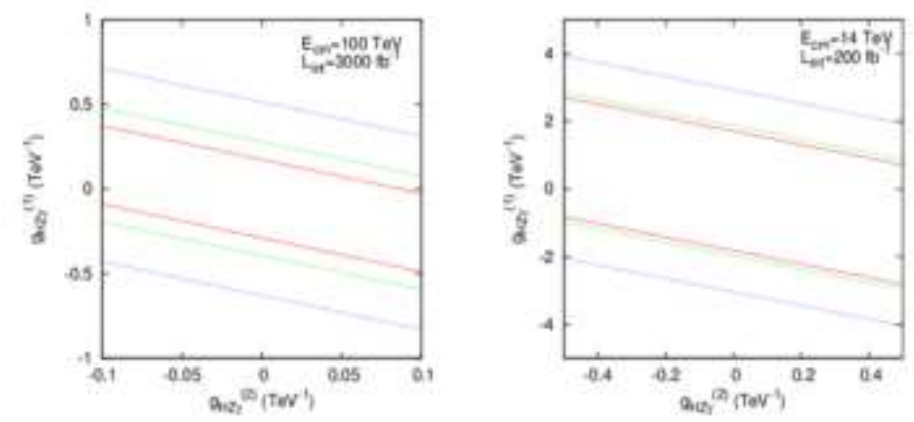

Figure 9. The 95\% C.L. sensitivity constraints on $g_{H Z \gamma}^{(2)}-g_{H Z \gamma}^{(1)}$ for $100 \mathrm{TeV}$ pp collider (left panel) and LHC (right panel). $\mathrm{H} \rightarrow \mathrm{Z} \gamma$ decay channel is considered as the signal. The solid line shows the bounds for the whole interval $0<\xi<1-\frac{m_{p}}{E_{p}}$. $t$ The dotted and dashed lines indicate the bounds for $0.015<\xi<0.15$ and $0.0015<\xi<0.5$, respectively.

\section{Conclusions}

We investigate the potential of the exclusive single Higgs boson production process at a future $100 \mathrm{TeV}$ proton-proton collider to examine non-standard $\mathrm{HZ} \gamma$ and $\mathrm{H} \gamma \gamma$ couplings. We get the sensitivity constraints on them and compare with the corresponding constraints of LHC. We observe from Figure 
6 that future $100 \mathrm{TeV}$ proton-proton collider with integrated luminosity of $L_{\text {int }}=3000 \mathrm{fb}^{-1}$ enable us to improve sensitivities on non-standard Higgs bounds by roughly a factor of 2.5 with respect to LHC. Also in Figure 7-Figure 9 one can simply see that at a future $100 \mathrm{TeV}$ proton-proton collider the sensitivity bounds on these couplings are roughly 4.5 times better than LHC limits. Thus, we conclude that a considerable improvement is achieved at a future $100 \mathrm{TeV}$ proton-proton collider to probe nonstandard Higgs constraints.

\section{References}

[1] Arkani-Hamed N., Han T., Mangano M., Wang L.T. 2016. Physics Opportunities of a $100 \mathrm{TeV}$ Proton-Proton Collider. Physics Reports, 652: 1-49.

[2] Mangano M.L., Zanderighi G., Aguilar Saavedra J.A., Alekhin S., Badger S., Bauer C.W., Becher T., Bertone V., Bonvini M., Boselli S., Bothmann E., et al. 2017. Physics at the FCC-hh, a 100 TeV pp collider. Cern Yellow Report, 3: 1-254.

[3] Contino R., Curtin D., Katz A., Mangano M.L., Panico G., Ramsey-Musolf M.J., Zanderighi G., Anastasiou C., Astill W., Bambhaniya G., et al. 2017. Physics at a $100 \mathrm{TeV}$ pp collider: Higgs and EW symmetry breaking studies. Cern Yellow Report, 3: 255-440.

[4] Aad G., Abajyan T., Abbott B., Abdallah J., Abdel Khalek S., Abdelalim A.A., Abdinov O., Aben R., Abi B., Abolins M., et al. 2012. Observation of a new particle in the search for the Standard Model Higgs boson with the ATLAS detector at the LHC. Physics Letters B, 716: 1-29.

[5] Chatrchyan S., Kharchatryan V., Sirunyan A.M., Tumasyan A., Adam W., Aguilo E., Bergauer T., Dragicevic M., Erö J., Fabjan C., et al. 2012. Observation of a new boson at a mass of 125 GeV with the CMS experiment at the LHC. Physics Letters B, 716: 30-61.

[6] Taheri Monfared S., Fayazbakhsh S., Mohammadi Najafabadi M. 2016. Exploring anomalous HZ $\gamma$ couplings in $\gamma$-proton collisions at the LHC. Physics Letters B, 762: 301-308.

[7] Cao O.H., Li G., Yan B., Zhang D.M., Zhang H. 2016. New measurement of antineutrino oscillation with the full detector configuration at Daya Bay. Physical Review Letters, 115: 11.

[8] Barr A.J., Dolan M.J., Englert C., Ferreira de Lima D.E., Spannowsky M. 2015. Future of High Energy Physics; The - Some Aspects. Journal of High Energy Physics, 1502: 016.

[9] Degrande C., Khoze V.V., Mattelaer O. 2016. Multi-Higgs-boson production in gluon fusion at $100 \mathrm{TeV}$. Physical Review D, 94: 085031.

[10] Fuks B., Kim J.H., Lee S.J. 2016. Multi-Higgs Production and Unitarity in Vector-Boson Fusion at Future Hadron Colliders. Physical Review D, 93: 035026.

[11] Baglio J., Djouadi A., Quevillon J. 2016. Prospects for Higgs physics at energies up to $100 \mathrm{TeV}$. Reports on Progress in Physics, 79: 116201.

[12] Papaefstathiou A., Sakurai K. 2016. Future of High Energy Physics; The - Some Aspects. Journal of High Energy Physics, 1602: 006.

[13] Albrow M.G., DeRoeck A., Khoze V.A., Lämsä J., Norbeck E., Onel Y., Orava R., Ryskin M.G. 2009. Forward physics with rapidity gaps at the LHC. Journal of Instrumentation, 4: P10001.

[14] Albrow M.G., Appleby R.B., Arneodo M., Atoian G., Azhgirey I.L., Barlow R., Bayshev I.S., Beaumont W., Bonnet L., Brandt A. 2009. The FP420 R\&D project: Higgs and New Physics with forward protons at the LHC. Journal of Instrumentation, 4: T10001.

[15] ATLAS Collaboration. 2011. Letter of Intent for the Phase-I Upgrade of the ATLAS Experiment. CERN-LHCC-2011-012.

[16] CMS and TOTEM collaboration. 2014. CMS-TOTEM Precision Proton Spectrometer. CERNLHCC-2014-021.

[17] Chatrchyan S., Kharchatryan V., Sirunyan A.M., Tumasyan A., Adam W., Aguilo E., Bergauer T., Dragicevic M., Erö J., Fabjan C., et al. 2012. Exclusive photon-photon production of muon pairs in proton-proton collisions at sqrt(s) $=7 \mathrm{TeV}$. Journal of High Energy Physics, 1201: 052.

[18] Chatrchyan S., Kharchatryan V., Sirunyan A.M., Tumasyan A., Adam W., Aguilo E., Bergauer T., Dragicevic M., Erö J., Fabjan C., et al. 2012. Search for exclusive or semi-exclusive $\gamma \gamma$ production and observation of exclusive and semi-exclusive $\mathrm{e}^{+} \mathrm{e}^{-}$production in pp collisions at $s \sqrt{ }=7 \mathrm{~s}=7 \mathrm{TeV}$. Journal of High Energy Physics, 1211: 080. 
[19] Chatrchyan S., Kharchatryan V., Sirunyan A.M., Tumasyan A., Adam W., Aguilo E., Bergauer T., Dragicevic M., Erö J., Fabjan C., et al. 2013. Differential branching fraction and angular analysis of the decay. Journal of High Energy Physics, 1307: 116.

[20] Khachatryan V., Sirunyan A.M., Tumasyan A., Adam W., Asilar E., Bergauer T., Brandstetter J., Brondolin E., Dragicevic M., Erö J. et al. 2016. Evidence for exclusive $\gamma \gamma \rightarrow \mathrm{W}+\mathrm{W}-$ production and constraints on anomalous quartic gauge couplings in pp collisions at $s \sqrt{ }=7$ and $8 \mathrm{TeV}$. Journal of High Energy Physics, 1608: 119.

[21] Aaboud M., Aad G., Abbott B., Abdallah J., Abdinov O., Abeloos B., Aben R., AbouZeid O.S., Abraham N.L., Abramowicz H. 2016. Measurement of exclusive gamma gamma -> W+Wproduction and search for exclusive Higgs boson production in pp collisions at root $\mathrm{s}=8 \mathrm{TeV}$ using the ATLAS detector. Physical Review D, 94: 032011.

[22] Abulencia A., Adelman J., Affolder T., Akimoto T., Albrow M.G., Ambrose D., Amerio S., Amidei D., Anastassov A., Anikeev K. et al. 2007. Observation of Exclusive Electron-Positron Production in Hadron-Hadron Collisions. Physical Review Letters, 98: 112001.

[23] Aaltonen T., Adelman J., Akimoto T., Albrow M.G., Alvarez Gonzalez B., Amerio S., Amidei D., Anastassov A., Annovi A., Antos J. et al. 2009. Search for Exclusive Z-Boson Production and

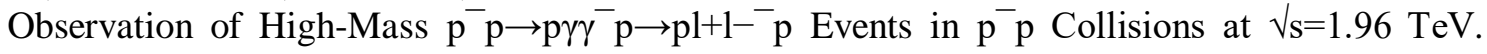
Physical Review Letters, 102: 222002.

[24] Aaltonen T., Adelman J., Akimoto T., Albrow M.G., Alvarez Gonzalez B., Amerio S., Amidei D., Anastassov A., Annovi A., Antos J. et al. 2009. Observation of exclusive charmonium production and $\gamma \gamma \rightarrow \mu+\mu$ - in $p \bar{p}$ collisions at $s=1.96 \mathrm{TeV}$. Physical Review Letters, 102: 242001 .

[25] Barger V., Ishida M., Keung W.Y. 2012. Total width of 125 GeV Higgs boson. Physical Review Letters, 108: 261801.

[26] Tasevsky M. 2015. AIP Conference Proceedings: Status of the AFP project in the ATLAS experiment, Cern, Geneva, Switzerland. 8 December 2014; Institute of Physics, Academy of Sciences of the Czech Republic: Na Slovance, Prague, Czech Republic, p. 4.

[27] Buchmuller W., Wyler D. 1986. Effective lagrangian analysis of new interactions and flavour conservation. Nuclear Physics B, 268: 621-653.

[28] Corbett T., Eboli O.J.P., Gonzalez-Fraile J., Gonzalez-Garcia M.C. 2012. Constraining anomalous Higgs interactions. Physical Review D, 86: 075013.

[29] Corbett T., Eboli O.J.P., Gonzalez-Fraile J., Gonzalez-Garcia M.C. 2013. Robust Determination of the Higgs Couplings: Power to the Data. Physical Review D, 87: 015022.

[30] Masso E., Sanz V. 2013. Limits on anomalous couplings of the Higgs boson to electroweak gauge bosons from LEP and the LHC. Physical Review D, 87: 033001.

[31] De Rujula A., Gavela M.B., Hernandez P., Masso E. 1992. The Selfcouplings of vector bosons: Does LEP-1 obviate LEP-2. Nuclear Physics B, 384: 3-58.

[32] Hagiwara K., Ishihara S., Szalapski R., Zeppenfeld D. 1993. Low energy effects of new interactions in the electroweak boson sector. Physical Review D, 48: 2182-2203.

[33] Gonzalez-Garcia M.C. 1999. Anomalous Higgs Couplings. International Journal of Modern Physics A, 14: 3121-3156.

[34] Leung C.N., Love S.T., Rao S.Z. 1986. Low-energy manifestations of a new interactions scale: Operator analysis. Physics C, 31: 433-437.

[35] Donoghue J.F., Golowich E., Holstein B.R. 1992. Dynamics of the Standard Model. Edited by Cambridge University Press, UK, 201.

[36] Farina M., Grossman Y., Robinson D.J. 2015. Probing CP Violation in $h \rightarrow Z \gamma$ with Background Interference. Physical Review D, 92: 073007. 